\title{
Normal early development in siblings with novel compound heterozygous variants in ASPM
}

\author{
Taro Moriwaki ${ }^{1}$, Narutoshi Yamazaki ${ }^{2}$, Tetsumin So ${ }^{3}$, Motomichi Kosuga ${ }^{1,2}$, Osamu Miyazaki ${ }^{4}$, Yoko Narumi-Kishimoto ${ }^{5}$, \\ Tadashi Kaname ${ }^{6}$, Gen Nishimura ${ }^{7}$, Torayuki Okuyama ${ }^{2}$ and Yasuyuki Fukuhara ${ }^{1}$
}

\begin{abstract}
Autosomal recessive primary microcephaly 5 (MCPH5) is caused by pathogenic variants in ASPM. Using whole-exome sequencing, we diagnosed two siblings with MCPH5. A known pathogenic variant (NM_018136.4: c.9697C > T, p. $($ Arg3233*)) and a novel pathogenic variant (c.1402_1406del, p.(Asn468Serfs*2)) of ASPM were identified in affected siblings with normal intelligence. Their pathogenic variants were not located in the critical regions of ASPM, but the relationship between the genotypes and their normal intelligence was unclear.
\end{abstract}

Microcephaly is an anthropological/descriptive sign rather than a specific diagnosis and is used to denote a significant reduction in the occipital-frontal circumference (OFC) when compared with the age-matched peer group ${ }^{1}$. Based on the etiology, microencephaly can be classified as primary (genetic) or secondary (nongenetic or environmental) ${ }^{2}$. Primary microcephaly is characterized by the prenatal onset of abnormal brain growth, resulting in a reduced brain volume (OFC $\geq 2$ standard deviations (SDs) ${ }^{3}$ or $3 \mathrm{SDs}^{4}$ below the age- and sex-matched means at birth). Most cases of primary microcephaly show an autosomal recessive mode of inheritance, and 18 microcephaly primary hereditary (MCPH; MIM \#251200) loci have been mapped to date (termed MCPH1-MCPH18) ${ }^{5}$. These genes affect not only cell cycle regulation but also DNA repair ${ }^{6,7}$. MCPH5 (MIM \#605481) is the most frequently reported type, accounting for up to $40 \%$ of cases in both consanguineous and nonconsanguineous families ${ }^{8}$. Microcephaly patients may have mild-to-severe mental retardation, short stature, seizures, or hereditary hearing $\operatorname{loss}^{9}$. The phenotypes among MCPH1-MCPH18 are quite

\footnotetext{
Correspondence: Yasuyuki Fukuhara (fukuhara-ys@ncchd.go.jp) 'Division of Medical Genetics, National Center for Child Health and Development, 2-10-1 Okura, Setagaya-ku, Tokyo 157-8535, Japan 2 Department of Clinical Laboratory Medicine, National Center for Child Health and Development, 2-10-1 Okura, Setagaya-ku, Tokyo 157-8535, Japan Full list of author information is available at the end of the article.
}

similar; therefore, exome sequencing is required for a precise diagnosis. Most patients with $\mathrm{MCPH} 5$ have been reported from the Middle East, and in East Asia, MCPH5 is rare, with just one sporadic case reported from Japan ${ }^{3}$. We herein report normal early development in a pair of Korean siblings with MCPH5 who had compound heterozygous ASPM (MIM\#605481) pathogenic variants that included a novel deletion.

The primary patient was a 3-year-old girl who had been born in Korea as the first live-born child of nonconsanguineous Korean parents. She had no familial history of microcephaly. She was suspected of having agenesis of the corpus callosum on fetal ultrasound. She was born at 39 weeks and 6 days of gestation. Her birth weight was $2940 \mathrm{~g}(-0.2 \mathrm{SD})$, her length was $47 \mathrm{~cm}$ $(-0.7 \mathrm{SD})$, and her OFC was $30 \mathrm{~cm}(-2.0 \mathrm{SD})$. She was referred to our hospital at 10 months because her parents moved to Japan for business. She had microcephaly and craniofacial dysmorphic features, including trigonocephaly and a small, sloped forehead. Her anterior fontanelle had already closed. Brain magnetic resonance imaging (MRI) and computed tomography (CT) revealed metopic suture craniosynostosis, which caused her trigonocephaly. Her brain had a simplified gyral pattern. In addition, significant small frontal lobes, small pons, and enlarged lateral ventricles were observed. Cerebellar hypoplasia was not observed. There was also a small 
arachnoid cyst in her left middle cranial fossa (Fig. 1). Developmental delay had not been noted since her infantile period. She held her head up steadily at 3 months, rolled over at 5 months, crawled at 11 months and walked alone at 1 year of age. At 10 months old, the Japanese Kyodaishiki developmental schedule showed that her intelligence quotient was 93 (motor func tion: 98, cognitive function: 89 , verbal function: 89 ). She is now 3 years old, and her body weight is $12.2 \mathrm{~kg}(-0.8 \mathrm{SD})$, her height is $89.5 \mathrm{~cm}(-1.2 \mathrm{SD})$, and her OFC is $40.5 \mathrm{~cm}$ $(-4.7 \mathrm{SD})$. Standard karyotyping showed $46, \mathrm{XX}$, and an array comparative genomic hybridization chromosomal test revealed no pathogenic deletions or duplications. We performed next-generation sequencing to determine the etiology of her microcephaly with informed consent from her parents. Genomic DNA was extracted from the peripheral blood of the patient and from her parents. The ethics committees of the National Center for Child Health and Development approved this study.

Her mother became pregnant while the genetic analysis for the primary patient was being performed. Fetal ultrasound revealed that the fetus was a male, and he also had microcephaly. The male patient was born at 38 weeks of gestation in Korea after his mother returned to her hometown. His birth weight was $2710 \mathrm{~g}(-0.7 \mathrm{SD})$, his length was $45.5 \mathrm{~cm}(-1.7 \mathrm{SD})$, and his OFC was $31.5 \mathrm{~cm}$ $(-1.3 \mathrm{SD})$. He was admitted to the neonatal intensivecare unit because he had microcephaly and transient tachypnea of the newborn (TTN). However, he recovered from the TTN quickly and finished all of his checkups, and he was able to be discharged at 10 days old. The brain MRI and CT findings were similar to his sister's findings and suggested microcephaly. Metopic suture craniosynostosis, a simplified gyral pattern and small frontal lobes were noted. He also had a small pons but no lateral ventricle enlargement. Cerebellar hypoplasia was not observed, just as in his sister (Fig. 1). At 3 months old, his body weight was $6.93 \mathrm{~kg}(+0.7 \mathrm{SD})$, his height was $59.8 \mathrm{~cm}(-0.7 \mathrm{SD})$, and his OFC was $35 \mathrm{~cm}(-2.9 \mathrm{SD})$. At 7 months old, his intelligence quotient was 102 (motor function: 93, cognitive function: 109, verbal function: 97). His sister's pathogenic variants of $A S P M$ had already been detected; therefore, his DNA was also analyzed using Sanger sequencing (Fig. 2).

Regarding the methods, genomic DNA was extracted from the peripheral blood using a QIAamp DNA Mini kit (Qiagen GmbH, Hilden, Germany). Whole-exome sequencing and analyses were performed as described previously $^{10}$. In brief, the SureSelect Human All Exon V6 kit (Agilent Technology, Santa Clara, CA, USA) was used for exon capture, and a HiSeq2500 system (Illumina, San Diego, CA, USA) with 101-bp paired-end reads was used for sequencing. Reads were aligned to GRCh37 using the Burrows-Wheeler Aligner (http://biobwa.sourceforge.net/). Variants were called using the GATK Unified Genotyper and ANNOVAR (http://annover.openbioinfomatics.org/ en/latest/). Detected $A S P M$ variants were validated by Sanger sequencing.

These analyses detected two different truncating pathogenic variants of $A S P M$ in the sister first and then
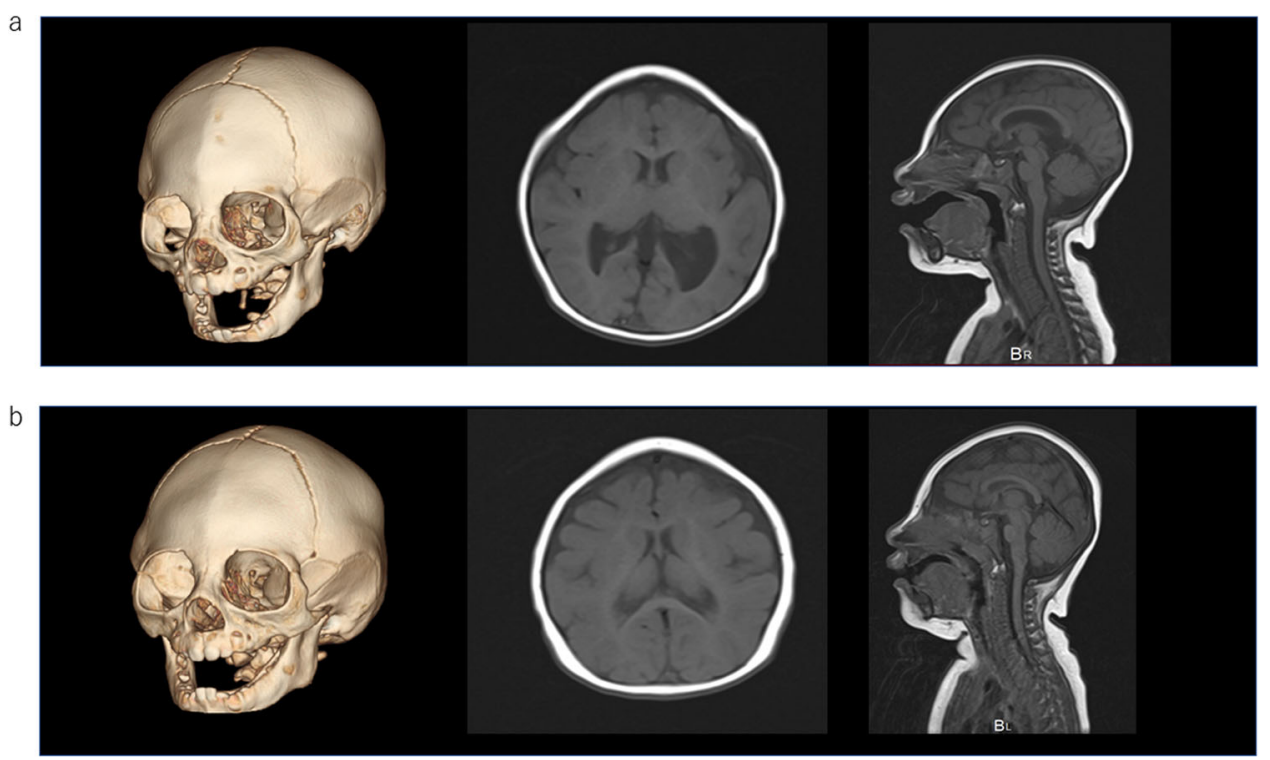

Fig. 1 Brain imaging. a The older sister at 2 years of age. Three-dimensional $C T$ (left), axial (middle), and sagittal (right) T1-weighted MRI. Metopic suture craniosynostosis led to trigonocephaly. Significant small frontal lobes, small pons, and enlarged lateral ventricles were observed. Cerebellar hypoplasia was not observed. $\mathbf{b}$ The younger brother at 6 months of age. The findings were similar to his sister's, but he did not have lateral ventricle enlargement. Cerebellar hypoplasia was not observed, just as in his sister. 


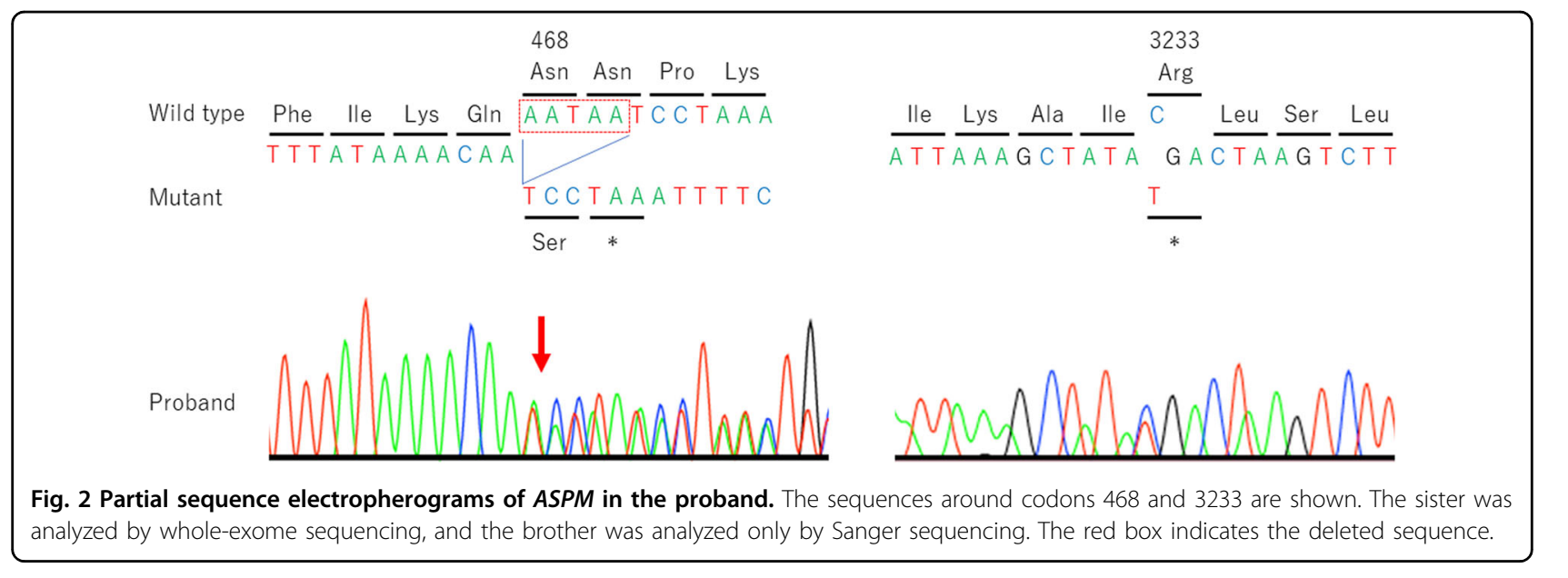

brother, NM_018136.4: c.1402_1406del, p.(Asn468Serfs*2) and c.9697C > T, p. (Arg3233*). Her father was a heterogeneous carrier of c.1402_1406del, p.(Asn468Serfs*2), and her mother was a carrier of c.9697C $>$ T, p. $\left(\operatorname{Arg} 3233^{*}\right)$, suggesting that the children had these pathogenic variants in a compound heterozygous state. The variant c.1402_1406del, p.(Asn468Serfs*2) was novel and not registered in various databases, such as HGVD, 1000 Genomes data, ExAC, gnomAD, and iJGVD. Mutation Taster, a prediction program, estimated the variant to be disease causing. In addition, the variant was classified as pathogenic according to ACMG guidelines. The variant c.9697C > T, p. (Arg3233*) was recurrent in MCPH5 patients. It was also classified as pathogenic according to ACMG guidelines. Both variants were estimated to result in mRNA decay because both premature stop codons are further than 50 nucleotides upstream of the last exon-exon junction.

The siblings have craniosynostosis of metopic suture and trigonocephaly in common, and these factors are associated with their similar facial appearance. As in previous reports ${ }^{11}$, they also had a simplified gyral pattern, small frontal lobes, and small pons. The sister had ventricular enlargement, but not the brother. Neither had a small cerebellar vermis. The sister's arachnoid cyst in the left middle cranial fossa was thought to have developed accidentally. MCPH5 patients usually have mild-to-severe intellectual disability ${ }^{11}$. However, developmental tests revealed the daughter's intelligence quotient to be 93 (motor function: 98, cognitive function: 89 , verbal function: 89 ) at 10 months old and the son's intelligence quotient to be 102 (motor function: 93, cognitive function: 109, verbal function: 97) at 7 months old, showing no developmental delay, at least during early infancy. Previously, a patient with normal motor development was reported ${ }^{12}$. Language development is thought to start being delayed when patients are older than 3 years of age ${ }^{13}$. Of note, most patients have been reported to have hyperactivity and/or speech problems ${ }^{11}$; therefore, we need to keep monitoring these siblings to determine whether they properly acquire the ability to learn and use language with age. ASPM pathogenic variants were reported to spread throughout the coding sequence of the gene, with no obvious genotype-phenotype correlation ${ }^{8}$. The ASPM protein forms a complex with another protein linked to microcephaly: the microtubule-severing ATPase katanin. The ASPM/katanin complex controls microtubule disassembly at spindle poles, and its misregulation can lead to microcephaly ${ }^{12}$. The ASPM protein contains an $\mathrm{N}$-terminal major sperm protein domain, a long unstructured region, calponin homology domains that can bind microtubules, multiple isoleucine-glutamine (IQ) motifs and a C-terminal HEAT repeat region ${ }^{12}$. The katanin $\mathrm{p} 60 \mathrm{~N} / \mathrm{p} 80 \mathrm{C}$ heterodimer interacts with the ASPM protein through its unstructured region, which contains several conserved peptide repeats ${ }^{14}$. The pathogenic variants (c.1402_1406del, p.(Asn468Serfs*2) and c. $\left.9697 \mathrm{C}>\mathrm{T}, \mathrm{p} .\left(\operatorname{Arg} 3233^{*}\right)\right)$ are located in the IQ motifs and C-terminal HEAT repeat region, respectively, which may explain why our patients had normal intelligence. However, Okamoto et $\mathrm{al}^{3}$ reported a Japanese patient who had ASPM pathogenic variants in exons 18 and 24 and showed severe intellectual disability. In addition, Mohamed's 21 Egyptian patients had a high proportion of consanguinity and consequently included a high percentage of patients with homozygous $A S P M$ pathogenic variants ${ }^{11}$. Notably, the patients with homozygous variants in exon 3 , where the $\mathrm{CH}$ domains are located, showed severe intellectual disability, and those with homozygous variants in exons $17,18,21$, and 23 , where the functional importance is unclear, also showed severe intellectual disability ${ }^{11}$. Furthermore, even among the pedigree of exon 17, intellectual 
disability among patients ranged from severe to mild ${ }^{11}$. Given these findings, it is difficult to wholly attribute the normal intelligence of our patients to their genotype.

\section{HGV database}

The relevant data from this Data Report are hosted at the Human Genome Variation Database at https://doi.org/10.6084/m9.figshare.hgv.2801

\section{Acknowledgements}

We thank Ms. Meiko Takeshita for her expert technical assistance.

\section{Author details}

'Division of Medical Genetics, National Center for Child Health and Development, 2-10-1 Okura, Setagaya-ku, Tokyo 157-8535, Japan. ${ }^{2}$ Department of Clinical Laboratory Medicine, National Center for Child Health and Development, 2-10-1 Okura, Setagaya-ku, Tokyo 157-8535, Japan. ${ }^{3}$ Division of Critical Care Medicine, National Center for Child Health and Development, 210-1 Okura, Setagaya-ku, Tokyo 157-8535, Japan. ${ }^{4}$ Department of Radiology, National Center for Child Health and Development, 2-10-1 Okura, Setagaya-ku, Tokyo 157-8535, Japan. ${ }^{5}$ Medical Genome Center, National Center for Child Health and Development, 2-10-1 Okura, Setagaya-ku, Tokyo 157-8535, Japan. ${ }^{6}$ Department of Genome Medicine, National Center for Child Health and Development, 2-10-1 Okura, Setagaya-ku, Tokyo 157-8535, Japan. ${ }^{7}$ Center of Intractable Diseases, Saitama Medical University Hospital, Saitama, Japan

\section{Conflict of interest}

The authors declare that they have no conflict of interest.

\section{Publisher's note}

Springer Nature remains neutral with regard to jurisdictional claims in published maps and institutional affiliations.

Received: 19 September 2019 Revised: 4 December 2019 Accepted: 5 December 2019.

Published online: 6 January 2020

\section{References}

1. Adachi, Y. et al. Congenital microcephaly with a simplified gyral pattern: associated findings and their significance. AJNR Am. J. Neuroradiol. 32, 1123-1129 (2011)

2. Abdel-Salam, G. M., Halász, A. A. \& Czeizel, A. E. Association of epilepsy with different groups of microcephaly. Dev. Med. Child Neurol. 42 760-767 (2000).

3. Okamoto, N., Kohmoto, T., Naruto, T., Masuda, K. \& Imoto, I. Primary microcephaly caused by novel compound heterozygous mutations in ASPM. Hum. Genome Var. 5, 18015 (2018).

4. Woods, C. G., Bond, J. \& Enard, W. Autosomal recessive primary microcephaly (MCPH): a review of clinical, molecular, and evolutionary findings. Am. J. Hum. Genet 76, 717-728 (2005).

5. Naveed, M. et al. Comprehensive review on the molecular genetics of autosomal recessive primary microcephaly (MCPH). Genet. Res. (Camb.) 100, e7 (2018).

6. Bond, J. et al. ASPM is a major determinant of cerebral cortical size. Nat. Genet. 32, 316-320 (2002).

7. Faheem, M. et al. Molecular genetics of human primary microcephaly: an overview. BMC Med. Genomics. 8, S4 (2015).

8. Nicholas, A. K. et al. The molecular landscape of ASPM mutations in primary microcephaly. J. Med. Genet. 46, 249-253 (2009).

9. Darvish, H. et al. A clinical and molecular genetic study of 112 Iranian families with primary microcephaly. J. Med. Genet. 47, 823-828 (2010).

10. Sasaki, H. et al. Definitive diagnosis of mandibular hypoplasia, deafness, progeroid features and lipodystrophy (MDPL) syndrome caused by a recurrent de novo mutation in the POLD1 gene. Endocr. J. 65, 227-238 (2018).

11. Abdel-Hamid, M. S. et al. Molecular and phenotypic spectrum of ASPM-related primary microcephaly: identification of eight novel mutations. Am. J. Med. Genet. A 170, 2133-2140 (2016).

12. Desir, J., Cassart, M., David, P., Van Bogaert, P. \& Abramowicz, M. Primary microcephaly with ASPM mutation shows simplified cortical gyration with antero-posterior gradient pre- and post-natally. Am. J. Med. Genet. A 146A, 1439-1443 (2008).

13. Passemard, S. et al. Expanding the clinical and neuroradiologic phenotype of primary microcephaly due to ASPM mutations. Neurology $\mathbf{7 3}$, 962-969 (2009).

14. Jiang, K. et al. Microtubule minus-end regulation at spindle poles by an ASPMkatanin complex. Nat. Cell Biol. 19, 480-492 (2017). 